\title{
Capacity of the continuous-space electromagnetic channel
}

Michael A. Jensen

jensen@byu.edu

Jon W. Wallace

wall@ieee.org

Follow this and additional works at: https://scholarsarchive.byu.edu/facpub

Part of the Electrical and Computer Engineering Commons

\section{Original Publication Citation}

Jensen, M. A., and J. W. Wallace. "Capacity of the Continuous-Space Electromagnetic Channel." Antennas and Propagation, IEEE Transactions on 56.2 (28): 524-31

\section{BYU ScholarsArchive Citation}

Jensen, Michael A. and Wallace, Jon W., "Capacity of the continuous-space electromagnetic channel" (2008). Faculty Publications. 199.

https://scholarsarchive.byu.edu/facpub/199 


\title{
Capacity of the Continuous-Space Electromagnetic Channel
}

\author{
Michael A. Jensen, Fellow, IEEE, and Jon W. Wallace, Member, IEEE
}

\begin{abstract}
Constructing the capacity bound of a multiple-input multiple-output wireless system is often performed by assuming specified antenna configurations and a propagation environment and determining the signaling strategy which maximizes throughput. This paper extends this approach to further determine the optimal antenna characteristics which maximize the capacity for the propagation scenario, with the resulting capacity bound representing the ultimate maximum achievable value if optimal antenna design and signaling are used. In this approach, the spatially-continuous transmit currents and receive fields are represented using eigenfunctions of appropriate operators. It is shown that, except under certain conditions where array supergain solutions emerge, the capacity remains bounded for finite transmit power. The approach also shows how to limit supergain effects using practical constraints. Model problems and numerical computations are provided for different power constraints at the transmitter and noise characteristics at the receiver.
\end{abstract}

Index Terms-Information theory, multipath channels, multipleinput multiple-output (MIMO) systems.

\section{INTRODUCTION}

$\mathbf{T}$ HE large potential capacity available from multiple-input multiple-output (MIMO) wireless communication technology in multipath channels [1]-[4] has motivated the development of sophisticated space-time coding schemes designed to approach the theoretical capacity limits [5]-[7]. In most prior work on this topic, the "channel" consists of a fixed physical propagation environment and antenna array configuration, revealing that antenna radiation characteristics will influence the channel capacity [4], [8]. This influence of antenna physical characteristics suggests that capacity computed for fixed arrays does not represent a true upper bound on achievable performance for a specific electromagnetic channel and indicates that the antenna design can be optimized for maximizing the throughput.

Before a meaningful approach to antenna synthesis for maximizing MIMO throughput can be formulated, it is necessary to first understand the upper performance bound for a given

Manuscript received August 4, 2006; revised August 22, 2007. This work was supported in part by the National Science Foundation under Information Technology Grant CCF-0428004 and in part by the U. S. Army Research Office under the Multi-University Research Initiative (MURI) Grant W911NF-04-10224.

M. A. Jensen is with the Department of Electrical and Computer Engineering, Brigham Young University, Provo, UT 84602 USA (e-mail: jensen@ee.by.edu).

J. W. Wallace was with the Department of Electrical and Computer Engineering, Brigham Young University, Provo, UT 84602 USA. He is now with the School of Engineering and Science, Jacobs University Bremen, Bremen 28759, Germany (e-mail: wall@ieee.org).

Digital Object Identifier 10.1109/TAP.2007.915416 electromagnetic channel independent of the antenna configuration. Work on this topic has typically followed one of two possible approaches. For example, the foundational study in [9] as well as the follow-on papers in [10], [11] formulate the communication in terms of transmit currents and receive field weighting distributions. In contrast, the studies in [12], [13] pose the problem in terms of the transmit and receive mode radiation patterns. Furthermore, regardless of the approach taken, each prior study presents the ultimate results of the analysis in terms of subtly different physical characteristics of the communication (capacity, number and quality of the channel modes, number of spatial degrees of freedom, etc.). As a result, it is difficult to ascertain: 1) the fundamental differences in the approaches, if any and 2) the basic differences or similarities in the conclusions drawn.

This paper develops a general framework which includes both prior analysis approaches, revealing that they represent solutions to the problem under different assumptions or constraints. Because this paper examines these solutions under a common framework, the distinctions between the conclusions drawn are also clarified. Specifically, the formulation represents the transmit current and receive weighting functions in terms of basis functions consisting of eigenfunctions of different operators depending on the transmit power constraint and receive noise model assumed. Because the framework uses currents and receiver weights, it produces the optimal transmit and receive antenna distributions as well as their associated radiation patterns, physical characteristics that are useful for antenna synthesis approaches that may be based on the proposed technique. Example closed-form solutions for model problems and numerical solutions for more general problems are provided which offer insight into the behavior of the capacity bound for different scenarios.

\section{ANALYSIS FRAMEWORK}

Before formulating the capacity bound of the channels under consideration, we first provide a framework for describing the continuous-space communication channel. Throughout this discussion, narrow-band signals are assumed with sinusoidal steady-state variation $\left(e^{j \omega t}\right)$. Additionally, boldface uppercase and lowercase letters describe matrices (matrix $\mathbf{H}$ with $m n$th element $H_{m n}$ ) and column vectors (vector $\mathbf{h}$ with $m$ th element $\left.h_{m}\right)$, respectively.

Fig. 1 depicts an arbitrary propagation scenario, where the transmit and receive elements are confined to the volumes $\Delta V_{\mathrm{t}}$ and $\Delta V_{\mathrm{r}}$ respectively. Local coordinate systems are assumed for the transmit space $\left(\mathbf{r}_{\mathrm{t}}\right)$ and receive space $\left(\mathbf{r}_{\mathrm{r}}\right)$. A dyadic Green's function $\mathbf{G}_{\mathrm{rt}}$, whose characteristics depend on the propagation environment, relates the vector current distribution $\mathbf{j}\left(\mathbf{r}_{\mathrm{t}}\right)$ in the 


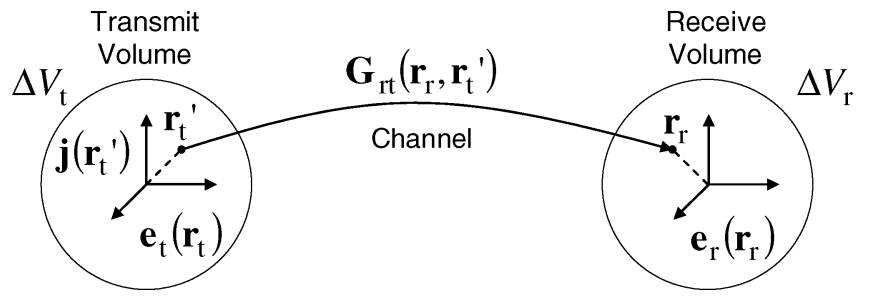

Fig. 1. Basic model for defining intrinsic capacity.

transmit volume to the signal-carrying electromagnetic fields in the receive volume. Assuming that a noise field $\mathbf{e}_{\eta}\left(\mathbf{r}_{\mathbf{r}}\right)$ exists in the receive volume, the total vector receive electric field can be written as [14]

$$
\mathbf{e}_{\mathrm{r}}\left(\mathbf{r}_{\mathrm{r}}\right)=\int_{\Delta V_{\mathrm{t}}} d \mathbf{r}_{\mathrm{t}}^{\prime} \mathbf{G}_{\mathrm{rt}}\left(\mathbf{r}_{\mathrm{r}}, \mathbf{r}_{\mathrm{t}}^{\prime}\right) \mathbf{j}\left(\mathbf{r}_{\mathrm{t}}^{\prime}\right)+\mathbf{e}_{\eta}\left(\mathbf{r}_{\mathrm{r}}\right)
$$

where the prime in $\mathbf{r}_{\mathrm{t}}^{\prime}$ denotes a source coordinate.

In assessing the behavior of this communication channel, it is necessary to understand the distribution (pattern) of the fields radiated by the transmit current. We examine this field behavior in the far-field with the transmitted electric field given as [14]

$$
\mathbf{e}_{\mathrm{t}}\left(\mathbf{r}_{\mathrm{t}}\right)=\frac{e^{-j k r_{\mathrm{t}}}}{r_{\mathrm{t}}} \int_{\Delta V_{\mathrm{t}}} d \mathbf{r}_{\mathrm{t}}^{\prime} \psi\left(\mathbf{r}_{\mathrm{t}}^{\prime}, \Omega_{\mathrm{t}}\right) \mathbf{j}\left(\mathbf{r}_{\mathrm{t}}^{\prime}\right)
$$

where $k$ is the wavenumber, $\Omega=(\theta, \phi)$ represents a solid-angle direction in the spherical coordinate frame with $\theta$ and $\phi$ denoting the elevation and azimuth angles, respectively, and

$$
\psi(\mathbf{r}, \Omega)=e^{j k(x \sin \theta \cos \phi+y \sin \theta \sin \phi+z \cos \theta)} .
$$

The power radiated by the transmit currents is then given as

$$
\begin{aligned}
p_{\mathrm{rad}} & =\lim _{r_{\mathrm{t}} \rightarrow \infty} \frac{1}{2 Z_{0}} \oint d \Omega_{\mathrm{t}} r_{\mathrm{t}}^{2} \mathbf{e}_{\mathrm{t}}^{\dagger}\left(\mathbf{r}_{\mathrm{t}}\right) \mathbf{e}_{\mathrm{t}}\left(\mathbf{r}_{\mathrm{t}}\right) \\
& =\int_{\Delta V_{\mathrm{t}}} d \mathbf{r}_{\mathrm{t} 1} \int_{\Delta V_{\mathrm{t}}} d \mathbf{r}_{\mathrm{t} 2} \mathbf{j}^{\dagger}\left(\mathbf{r}_{\mathrm{t} 1}\right) \frac{\Psi\left(\mathbf{r}_{\mathrm{t} 1}, \mathbf{r}_{\mathrm{t} 2}\right)}{2 Z_{0}} \mathbf{j}\left(\mathbf{r}_{\mathrm{t} 2}\right) \\
\Psi\left(\mathbf{r}_{\mathrm{t} 1}, \mathbf{r}_{\mathrm{t} 2}\right) & =\oint d \Omega_{\mathrm{t}} \psi^{*}\left(\mathbf{r}_{\mathrm{t} 1}, \Omega_{\mathrm{t}}\right) \psi\left(\mathbf{r}_{\mathrm{t} 2}, \Omega_{\mathrm{t}}\right)
\end{aligned}
$$

where $Z_{0}$ is the free-space intrinsic impedance, $\oint$ denotes integration over the unit sphere, $\{\cdot\}^{\dagger}$ represents a matrix conjugate transpose, and $\{\cdot\}^{*}$ is a conjugate.

If we now expand the transmit current using a set of basis functions $\boldsymbol{\tau}_{n}\left(\mathbf{r}_{\mathrm{t}}\right)$ and then project the received field in (1) onto a set of receive basis functions $\boldsymbol{\rho}_{m}\left(\mathbf{r}_{\mathbf{r}}\right)$, the result is

$$
\begin{aligned}
\mathbf{j}\left(\mathbf{r}_{\mathrm{t}}^{\prime}\right) & =\sum_{n} x_{n} \boldsymbol{\tau}_{n}\left(\mathbf{r}_{\mathrm{t}}^{\prime}\right) \\
y_{m} & =\int_{\Delta V_{\mathrm{r}}} d \mathbf{r}_{\mathrm{r}} \boldsymbol{\rho}_{m}^{\dagger}\left(\mathbf{r}_{\mathrm{r}}\right) \mathbf{e}_{\mathrm{r}}\left(\mathbf{r}_{\mathrm{r}}\right)=\sum_{n} H_{m n} x_{n}+\eta_{m} \\
H_{m n} & =\int_{\Delta V_{\mathrm{r}}} d \mathbf{r}_{\mathrm{r}} \int_{\Delta V_{\mathrm{t}}} d \mathbf{r}_{\mathrm{t}}^{\prime} \boldsymbol{\rho}_{m}^{\dagger}\left(\mathbf{r}_{\mathrm{r}}\right) \mathbf{G}_{\mathrm{rt}}\left(\mathbf{r}_{\mathrm{r}}, \mathbf{r}_{\mathrm{t}}^{\prime}\right) \boldsymbol{\tau}_{n}\left(\mathbf{r}_{\mathrm{t}}^{\prime}\right) \\
\eta_{m} & =\int_{\Delta V_{\mathrm{r}}} d \mathbf{r}_{\mathrm{r}} \boldsymbol{\rho}_{m}^{\dagger}\left(\mathbf{r}_{\mathrm{r}}\right) \mathbf{e}_{\eta}\left(\mathbf{r}_{\mathrm{r}}\right) .
\end{aligned}
$$

We now have a discrete (although possibly infinite-dimensional) representation of the communication channel that forms the basis for the analyses in the following sections.

While this formulation is general for any propagation Green's function, for the examples appearing in this paper we adopt a commonly-used plane-wave model for a multipath channel where

$$
\mathbf{G}_{\mathrm{rt}}\left(\mathbf{r}_{\mathrm{r}}, \mathbf{r}_{\mathrm{t}}\right)=\sum_{\ell=1}^{L} \mathbf{G}_{0 \ell} \beta_{\ell} \psi\left(\mathbf{r}_{\mathrm{r}}, \Omega_{\mathrm{r} \ell}\right) \psi\left(\mathbf{r}_{\mathrm{t}}, \Omega_{\mathrm{t} \ell}\right)
$$

which indicates the channel consists of $L$ plane waves with the $\ell$ th wave described by a complex gain $\beta_{\ell}$, departure angle $\Omega_{\mathrm{t} \ell}=\left(\theta_{\mathrm{t} \ell}, \phi_{\mathrm{t} \ell}\right)$ and arrival angle $\Omega_{\mathrm{r} \ell}=\left(\theta_{\mathrm{r} \ell}, \phi_{\mathrm{r} \ell}\right)$. The complex gain $\beta_{\ell}$ includes all path loss, absorption, and scattering loss encountered in the propagation channel, while the dyad $\mathbf{G}_{0 \ell}$ represents the channel polarization response and is characterized by unit Frobenius norm.

\section{TRaditional Channel Description}

We are now poised to compute the capacity of the continuousspace channel described in Section II. We begin by making some traditional assumptions about the channel to obtain a simple capacity expression. Since it is difficult if not impossible to determine the capacity bounds for general channel characteristics and transmit/receive volumes, we also demonstrate a simple numerical procedure that closely approximates the channel capacity bound.

\section{A. Capacity Formulation}

Following traditional discrete-space analyses of the MIMO channel where the sum of the squares of the transmit antenna current excitations is constrained [1], [2] we use in this continuous case the constraint

$$
\int_{\Delta V_{\mathrm{t}}} d \mathbf{r}_{\mathrm{t}}^{\prime} \mathrm{E}\left\{\left|\mathbf{j}\left(\mathbf{r}_{\mathrm{t}}^{\prime}\right)\right|^{2}\right\} \leq P_{T}
$$

where $\mathrm{E}\{\cdot\}$ represents an expectation and $P_{T}$ represents a constant that can be chosen based on maximum allowable current magnitude. If the basis functions $\boldsymbol{\tau}_{n}\left(\mathbf{r}_{\mathrm{t}}\right)$ are orthonormal for integration over the transmit volume, then the discretized version of this constraint resulting from substitution of (6) into (11) becomes

$$
\sum_{n} \mathrm{E}\left\{\left|x_{n}\right|^{2}\right\} \leq P_{T}
$$

which is a convenient form for analysis since it matches the traditional constraint used for capacity computation.

While it is not typically explicitly stated, most capacity formulations assume that the noise at the receiver is spatially white, which is appropriate for thermal noise generated in the receiver front-end. In the continuous representation, let $\mathbf{e}_{\eta}\left(\mathbf{r}_{\mathrm{r}}\right)$ be a spatially uncorrelated zero-mean complex Gaussian random process with

$$
\mathrm{E}\left\{\mathbf{e}_{\eta}\left(\mathbf{r}_{\mathrm{r}}\right) \mathbf{e}_{\eta}^{\dagger}\left(\mathbf{r}_{\mathrm{r}}^{\prime}\right)\right\}=\sigma_{\eta}^{2} \mathbf{I} \delta\left(\mathbf{r}_{\mathrm{r}}-\mathbf{r}_{\mathrm{r}}^{\prime}\right)
$$

where $\mathbf{I}$ is the identity matrix and $\delta(\cdot)$ is the Dirac delta function. If the receive basis functions are orthonormal with respect to 
integration over the receive volume, the noise samples $\eta_{m}$ in (9) are zero-mean complex Gaussian random variables with crosscovariance

$$
R_{\eta, m p}=\mathrm{E}\left\{\eta_{m} \eta_{p}^{*}\right\}=\sigma_{\eta}^{2} \delta_{m p}
$$

where $\delta_{m p}$ is a Kronecker delta, indicating spatially white receiver noise as desired.

Our task is therefore to find a satisfactory basis for the formulation. We assume that the channel operator in (1) is compact, which has the implication that bounded transmit currents lead to bounded received fields [15]. Any physically realistic channel Green's function $\mathbf{G}_{\mathrm{rt}}\left(\mathbf{r}_{\mathrm{r}}, \mathbf{r}_{\mathrm{t}}\right)$ will exhibit this property. Under this assumption, a convenient basis that provides the required orthonormality consists of the eigenfunctions $\boldsymbol{\rho}_{m}\left(\mathbf{r}_{\mathrm{r}}\right)$ and $\boldsymbol{\tau}_{m}\left(\mathbf{r}_{\mathrm{t}}\right)$ of the channel operator expressed in (8) [15]. The result of this choice is that $H_{m n}=0$ for $m \neq n$, with the channel eigenvalue $H_{m m}$ representing the voltage gain of the $m$ th orthogonal spatial sub-channel.

Since this formulation has decomposed the problem into independent channels with Gaussian noise, we can immediately write that the capacity for this infinite-dimensional problem is [16]

$$
C=\max _{R_{x, m m}} \sum_{m} \log _{2}\left[1+\frac{\left|H_{m m}\right|^{2} R_{x, m m}}{\sigma_{\eta}^{2}}\right]
$$

where $R_{x, m m}=\mathrm{E}\left\{\left|x_{m}\right|^{2}\right\}$ is the power allocated to the $m$ th sub-channel as computed by the water-filling solution under the constraint (12) to maximize the capacity [16].

This formulation is equivalent to that outlined in [9]-[11] (although explicit power constraints are not discussed in [9]). Using the channel eigenfunctions as the transmit and receive bases is natural since it provides the necessary orthonormality of the basis functions (which is required to arrive at the desired form for the transmit constraint and receiver noise) and is directly analogous to traditional discrete-space analyses of the MIMO capacity problem [1], [2]. Furthermore, this analysis reveals that the capacity for this traditional channel depends only on the eigenvalues $H_{m m}$ that depend on the transmit and receive volumes and the propagation environment. Provided that there are a finite number of non-zero gains $\left|H_{m m}\right|$ which have the same value, water-filling will select only a finite number of communication modes, leading to bounded capacity for finite $P_{T}$, as is verified by example scenarios in the following sections.

\section{B. Model Problem}

Application of this framework to a simplified problem illustrates the concepts discussed. Consider a line-of-sight (LOS) propagation channel with single-polarization fields. The scalar Green's function for this scenario is

$$
G_{\mathrm{rt}}\left(\mathbf{r}_{\mathrm{r}}, \mathbf{r}_{\mathrm{t}}^{\prime}\right)=\beta e^{-j k\left(x_{\mathrm{r}}-x_{\mathrm{t}}^{\prime}\right)}
$$

where $\beta$ is a complex scalar. We can immediately ascertain that the single scalar eigenfunction pair for this channel is [9]

$$
\tau_{1}\left(\mathbf{r}_{\mathrm{t}}\right)=\frac{e^{-j k x_{\mathrm{t}}}}{\sqrt{\Delta V_{\mathrm{t}}}} \quad \rho_{1}\left(\mathbf{r}_{\mathrm{r}}\right)=\frac{e^{-j k x_{\mathrm{r}}}}{\sqrt{\Delta V_{\mathrm{r}}}}
$$

with associated eigenvalue $H_{11}=\beta \sqrt{\Delta V_{\mathrm{r}} \Delta V_{\mathrm{t}}}$. Clearly, the capacity bound of this channel is completely determined by this single eigenvalue coupled with the noise power $\sigma_{\eta}^{2}$, which is precisely the expected result for the LOS far-field channel. We emphasize that larger volumes lead to increased capacity due to the increased spatial filtering ability associated with larger apertures.

\section{Numerical Approximation of Eigenfunctions}

The set of channels (and transmit/receive volumes) for which the channel operator eigenfunctions can be determined in closed form appears to be limited [9]. Therefore, when analyzing complicated multipath channel Green's functions or arbitrary transmit and receive volume shapes, we must resort to numerical solutions that provide a close approximation to these eigenfunctions.

In this approach, we express the continuous basis functions as a linear combination of sub-basis functions that span the relevant spaces in a limiting sense as the number of sub-basis functions becomes infinite, or

$$
\begin{aligned}
& \boldsymbol{\tau}_{n}\left(\mathbf{r}_{\mathrm{t}}\right)=\sum_{q} C_{q n} \tilde{\boldsymbol{\tau}}_{q}\left(\mathbf{r}_{\mathrm{t}}\right) \\
& \boldsymbol{\rho}_{m}\left(\mathbf{r}_{\mathrm{r}}\right)=\sum_{p} B_{p m} \tilde{\boldsymbol{\rho}}_{p}\left(\mathbf{r}_{\mathrm{r}}\right)
\end{aligned}
$$

where $\tilde{\boldsymbol{\tau}}$ and $\tilde{\boldsymbol{\rho}}$ are respectively orthonormal transmit and receive sub-basis functions for integration over the volume. Substitution of (18) into (8) gives

$$
\begin{aligned}
H_{m n}= & \int_{\Delta V_{\mathrm{r}}} d \mathbf{r}_{\mathrm{r}} \int_{\Delta V_{\mathrm{t}}} d \mathbf{r}_{\mathrm{t}}^{\prime}\left[\sum_{p} B_{p m} \tilde{\boldsymbol{\rho}}_{p}\left(\mathbf{r}_{\mathrm{r}}\right)\right]^{\dagger} \mathbf{G}_{r t}\left(\mathbf{r}_{\mathrm{r}}, \mathbf{r}_{\mathrm{t}}^{\prime}\right) \\
& \times \sum_{q} C_{q n} \tilde{\boldsymbol{\tau}}_{q}\left(\mathbf{r}_{\mathrm{t}}^{\prime}\right) \\
= & \sum_{p} \sum_{q} B_{p m}^{*} \widetilde{H}_{p q} C_{q n}=\left\{\mathbf{B}^{\dagger} \tilde{\mathbf{H}} \mathbf{C}\right\}_{m n}
\end{aligned}
$$

where we have used matrix notation for simplicity assuming we will ultimately restrict ourselves to a finite sub-basis set. For the expansions in (18) to represent approximations to the desired eigenfunctions, $\mathbf{H}$ must be diagonal. Representing $\widetilde{\mathbf{H}}$ in terms of its singular value decomposition (SVD) $\widetilde{\mathbf{H}}=\widetilde{\mathbf{U}} \widetilde{\mathbf{S}} \widetilde{\mathbf{V}}^{\dagger}$, we obtain $\mathbf{H}=\mathbf{B}^{\dagger} \widetilde{\mathbf{U}} \widetilde{\mathbf{S}} \widetilde{\mathbf{V}}^{\dagger} \mathbf{C}$. Assigning $\mathbf{C}=\widetilde{\mathbf{V}}$ and $\mathbf{B}=\widetilde{\mathbf{U}}$ yields $\mathbf{H}=\widetilde{\mathbf{S}}$ which is diagonal as desired. Also, since $\tilde{\boldsymbol{\tau}}$ and $\tilde{\boldsymbol{\rho}}$ are orthonormal and $\widetilde{\mathbf{U}}$ and $\widetilde{\mathbf{V}}$ are unitary, this assignment ensures that $\boldsymbol{\tau}$ and $\boldsymbol{\rho}$ are also orthonormal. 


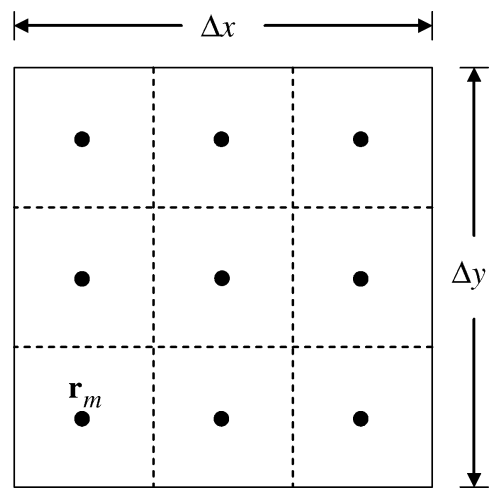

Fig. 2. Top view of an array aperture showing the regions and centers of support of the sub-basis functions used for numerical implementation of the capacity framework for $N=3$.

Under this representation, we use (18) in (6) to obtain

$$
\mathbf{j}\left(\mathbf{r}_{\mathrm{t}}^{\prime}\right)=\sum_{q} \underbrace{\left(\sum_{n} C_{q n} x_{n}\right)}_{\tilde{x}_{q}} \tilde{\boldsymbol{\tau}}_{q}\left(\mathbf{r}_{\mathrm{t}}^{\prime}\right)
$$

where $\tilde{x}_{q}$ is the complex weight on the $q$ th sub-basis function. Since $\tilde{\mathbf{x}}=\mathbf{C x}=\widetilde{\mathbf{V}} \mathbf{x}$, the covariance of $\tilde{\mathbf{x}}$ is $\widetilde{\mathbf{R}} x=\widetilde{\mathbf{V}} \mathbf{R}_{x} \widetilde{\mathbf{V}}^{\dagger}$. Solving this for $\mathbf{R}_{x}$ and placing the result into (15) yields

$$
\begin{aligned}
C & =\max _{\widetilde{\mathbf{R}}_{x}} \log _{2} \operatorname{det}\left[\frac{\widetilde{\mathbf{S}} \widetilde{\mathbf{V}}^{\dagger} \widetilde{\mathbf{R}}_{x} \widetilde{\mathbf{V}} \widetilde{\mathbf{S}}}{\sigma_{\eta}^{2}}+\mathbf{I}\right] \\
& =\max _{\widetilde{\mathbf{R}}_{x}} \log _{2} \operatorname{det}\left[\frac{\widetilde{\mathbf{H}} \widetilde{\mathbf{R}}_{x} \widetilde{\mathbf{H}}^{\dagger}}{\sigma_{\eta}^{2}}+\mathbf{I}\right]
\end{aligned}
$$

where we have used $\widetilde{\mathbf{H}}=\widetilde{\mathbf{U}} \widetilde{\mathbf{S}} \widetilde{\mathbf{V}}^{\dagger}$ with $\widetilde{\mathbf{U}}$ unitary. Equation (12) can also be represented as $\operatorname{Tr}\left(\widetilde{\mathbf{R}}_{x}\right) \leq P_{T}$, where $\operatorname{Tr}(\cdot)$ represents the trace. These results reveal that the capacity can be obtained by the water-filling solution using the transfer matrix $\widetilde{\mathbf{H}}$ and covariance $\widetilde{\mathbf{R}}_{x}$ for the sub-basis functions.

We can demonstrate application of this technique for a practical scenario. We assume a single electromagnetic polarization with propagation confined to the horizontal plane $\theta=$ $\pi / 2$ in all expressions). The multipath propagation channel described by (10) will be used with multipath parameters drawn from statistical distributions that have been found accurate for representing indoor propagation environments [17], [18]. Because this represents a two-dimensional environment, the basis functions for purposes of this example computation can also be two-dimensional. The transmit and receive apertures are assumed to be rectangular with side lengths $\Delta x$ and $\Delta y$ in the $x$ and $y$ dimensions, respectively. An appropriate set of sub-basis functions is

$$
\begin{array}{ll}
\tilde{\rho}_{m}(\mathbf{r}) \\
\tilde{\tau}_{m}(\mathbf{r})
\end{array}= \begin{cases}\frac{N}{\sqrt{\Delta x \Delta y}}=\frac{1}{b}, & \left|x-x_{m}\right|<\frac{\Delta x}{2 N} \\
0, & \left|y-y_{m}\right|<\frac{\Delta y}{2 N} \\
0, & \text { otherwise, }\end{cases}
$$

where $N$ is the number of subdivisions in $x$ and $y$ and $\mathbf{r}_{m}=$ $\left(x_{m}, y_{m}\right)$ defines the support region center for the $m$ th basis function. Fig. 2 illustrates one of these areas along with the region of support of the basis functions for $N=3$.

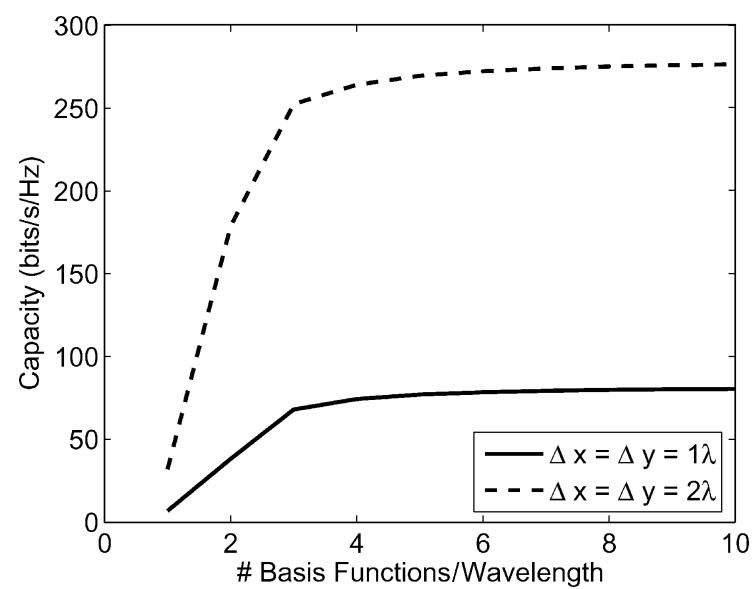

Fig. 3. Numerically computed capacity versus the number of sub-basis elements per dimension per wavelength for the traditional channel model for two different aperture sizes. For fine sampling, the capacity approaches an upper bound.

Using (19), the channel matrix for the sub-basis functions has elements

$$
\widetilde{H}_{m n}=b^{2} \sum_{\ell=1}^{L} \beta_{\ell} \gamma\left(\mathbf{r}_{\mathrm{r} m}, \Omega_{\mathrm{r} \ell}\right) \gamma\left(\mathbf{r}_{\mathrm{t} n}, \Omega_{\mathrm{t} \ell}\right)
$$

where $\theta_{\mathrm{t} \ell}=\theta_{\mathrm{r} \ell}=\pi / 2$ and

$$
\gamma(\mathbf{r}, \Omega)=\psi(\mathbf{r}, \Omega) \frac{\sin (\zeta \Delta x \cos \phi)}{\zeta \Delta x \cos \phi} \frac{\sin (\zeta \Delta y \sin \phi)}{\zeta \Delta y \sin \phi}
$$

with $\zeta=k \sin \theta / 2 N$.

We assume identical transmit and receive areas with either $\Delta x=\Delta y=1 \lambda$ or $2 \lambda$, where $\lambda$ is the free-space wavelength. The channel is formed from a single realization of the statistical path-based channel model consisting of 78 different paths grouped into six different angular clusters using the parameters outlined in [17]. Using a single sub-basis function in the transmit and receive volumes $(N=1)$, we compute the channel gain $\widetilde{H}_{11}$ and define the signal-to-noise ratio (SNR) as

$$
\mathrm{SNR}=\frac{P_{T}\left|\widetilde{H}_{11}\right|^{2}}{\sigma_{\eta}^{2}}
$$

where $P_{T}$ is the bound used in the power constraint (11) which is arbitrarily set to $P_{T}=1$ for this computation. The value of $\sigma_{\eta}^{2}$ is then chosen to obtain an SNR of $20 \mathrm{~dB}$, and this value is held constant as the number of sub-basis functions increases.

Fig. 3 shows the capacity obtained from this computation as a function of the number of basis functions per wavelength for the two aperture dimensions. As can be seen, the capacity approaches an upper bound as the number of sub-basis functions increases. In fact, when the sub-basis function density exceeds six, the capacity bound is relatively insensitive to the addition of basis functions, indicating that this density of sub-basis functions is adequate for describing the channel behavior for this scenario. This result is key, in that it numerically confirms the postulation that the achievable capacity for this scenario remains 


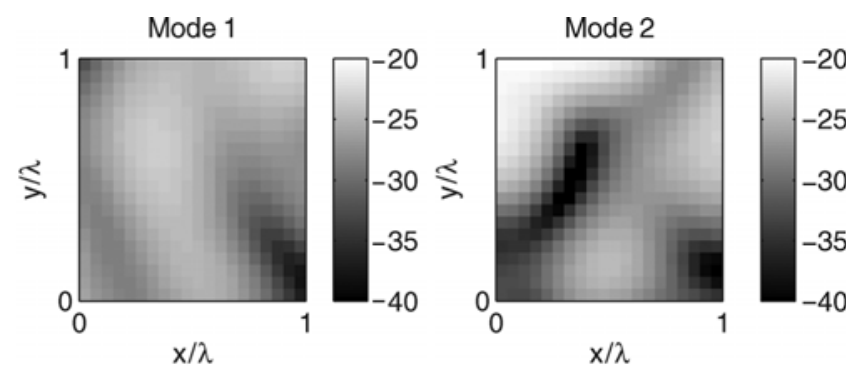

(a)
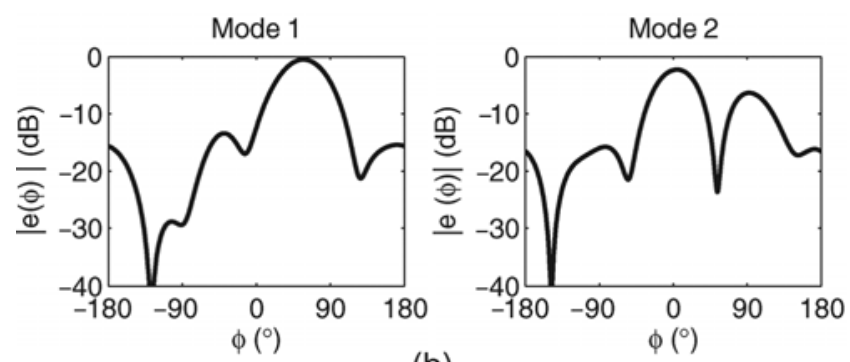

(b)

Fig. 4. Numerically computed (a) currents in $\mathrm{dB}$ and (b) radiation patterns for the dominant two transmit eigenfunctions for the case considered in Fig. $3(\Delta x=\Delta y=1 \lambda)$.

bounded. The plot also reveals the strong dependence of the capacity on the aperture size, as expected.

As mentioned in the introduction, an advantage of the framework used here is that it produces the transmit currents and receive weights as well as the associated radiation patterns [through the use of (2)]. Fig. 4 plots the currents and patterns for the dominant (associated with the largest channel eigenvalues) two transmit eigenfunctions for the case considered in Fig. 3 for $N=20$ and $\Delta x=\Delta y=1 \lambda$. Antennas capable of providing or closely approximating these currents and radiation patterns represent the optimal antenna element designs for this scenario. Certainly, a practical antenna design must provide good performance over a variety of environments rather than a single propagation scenario. However, coupling of this capability with some type of stochastic analysis may provide a method for designing antennas which are appropriate for deployment in practical systems. This remains a topic for future research.

\section{Modified ChanNel Description}

The analysis presented in Section III neglects some key physical issues that must be considered. Specifically, examination of (4) clearly indicates that the constraint in (11) does not actually limit the amount of power radiated by the transmit currents. Similarly, the model of Section III does not explore the implications of noise generated external to the receiver. This section shows that incorporation of these issues dramatically changes the formulation as well as the final conclusions.

\section{A. Capacity Formulation}

We now modify our transmit constraint to limit the total radiated power as computed in (4), or $\mathrm{E}\left\{p_{\mathrm{rad}}\right\} \leq P_{T}$. To start the formulation, consider writing the radiated power from (4) as

$$
\begin{aligned}
& p_{\text {rad }}=\sum_{n, q} x_{n}^{*} A_{n q} x_{q} \\
& A_{n q}=\int_{\Delta V_{\mathrm{t}}} d \mathbf{r}_{\mathrm{t} 1} \int_{\Delta V_{\mathrm{t}}} d \mathbf{r}_{\mathrm{t} 2} \boldsymbol{\tau}_{n}^{\dagger}\left(\mathbf{r}_{\mathrm{t} 1}\right) \frac{\Psi\left(\mathbf{r}_{\mathrm{t} 1}, \mathbf{r}_{\mathrm{t} 2}\right)}{2 Z_{0}} \boldsymbol{\tau}_{q}\left(\mathbf{r}_{\mathrm{t} 2}\right) .
\end{aligned}
$$

Computing the capacity for this infinite-dimensional problem ultimately requires that we express the power constraint in a form equivalent to that in (12). This can be accomplished by letting $\boldsymbol{\tau}_{n}\left(\mathbf{r}_{\mathrm{t}}\right)$ be the eigenfunctions of the operation in (27) so that $A_{n q}=0$ for $n \neq q$. Since the operator in (27) is self-adjoint, the eigenvalues $A_{n n}$ will be real. We can therefore define $\hat{x}_{n}=\sqrt{A_{n n}} x_{n}$ so that

$$
p_{\text {rad }}=\sum_{n}\left|\hat{x}_{n}\right|^{2} .
$$

We encounter a similar situation for noise generated external to the receive sub-system. In this case we assume that a noise field $\mathbf{n}\left(\Omega_{\mathrm{r}}\right)$ impinges on the receive volume which is modeled as a zero-mean complex Gaussian random process with

$$
\mathrm{E}\left\{\mathbf{n}\left(\Omega_{\mathrm{r}}\right) \mathbf{n}^{\dagger}\left(\Omega_{\mathrm{r}}^{\prime}\right)\right\}=p_{\eta}\left(\Omega_{\mathrm{r}}\right) \mathbf{I} \delta\left(\Omega_{\mathrm{r}}-\Omega_{\mathrm{r}}^{\prime}\right)
$$

where $p_{\eta}\left(\Omega_{\mathrm{r}}\right)$ represents the noise power angular spectrum. The received noise is

$$
\mathbf{e}_{\eta}\left(\mathbf{r}_{\mathrm{r}}\right)=\oint d \Omega_{\mathrm{r}} \psi\left(\mathbf{r}_{\mathrm{r}}, \Omega_{\mathrm{r}}\right) \mathbf{n}\left(\Omega_{\mathrm{r}}\right)
$$

leading to the expression

$$
\begin{aligned}
\mathrm{E}\left\{\mathbf{e}_{\eta}\left(\mathbf{r}_{\mathrm{r} 1}\right) \mathbf{e}_{\eta}^{\dagger}\left(\mathbf{r}_{\mathrm{r} 2}\right)\right\} & =\mathbf{I} \oint d \Omega_{\mathrm{r}} \psi\left(\mathbf{r}_{\mathrm{r} 1}, \Omega_{\mathrm{r}}\right) \psi^{*}\left(\mathbf{r}_{\mathrm{r} 2}, \Omega_{\mathrm{r}}\right) p_{\eta}\left(\Omega_{\mathrm{r}}\right) \\
& =\mathbf{I} \Psi_{\eta}\left(\mathbf{r}_{\mathrm{r} 1}, \mathbf{r}_{\mathrm{r} 2}\right) .
\end{aligned}
$$

Constructing $\eta_{m}$ as in (9), the output noise has cross-covariance $R_{\eta, m p}=\mathrm{E}\left\{\eta_{m} \eta_{p}^{*}\right\}$ given by

$$
R_{\eta, m p}=\int_{\Delta V_{\mathrm{r}}} d \mathbf{r}_{\mathrm{r} 1} \int_{\Delta V_{\mathrm{r}}} d \mathbf{r}_{\mathrm{r} 2} \boldsymbol{\rho}_{m}^{\dagger}\left(\mathbf{r}_{\mathrm{r} 1}\right) \Psi_{\eta}\left(\mathbf{r}_{\mathrm{r} 1}, \mathbf{r}_{\mathrm{r} 2}\right) \boldsymbol{\rho}_{p}\left(\mathbf{r}_{\mathrm{r} 2}\right) .
$$

Once again, evaluating the properties of the capacity for this infinite-dimensional case requires that we have spatially uncorrelated noise. This is satisfied if $\boldsymbol{\rho}_{m}\left(\mathbf{r}_{\mathrm{r}}\right)$ represents the $m$ th eigenfunction of (32) so that $R_{\eta, m p}=0$ for $m \neq p$. We note that if the noise is isotropic $\left(p_{\eta}\left(\Omega_{\mathrm{r}}\right)=1\right)$, the transmit and receive eigenfunctions are identical.

These transmit and receive basis functions are appropriate as they allow simple formulation of the communication model. Specifically, constructing the signal $\hat{y}_{m}=y_{m} / \sqrt{R_{\eta, m m}}$ and using $\hat{x}_{n}=\sqrt{A_{n n}} x_{n}$ in the expressions of (6)-(9) leads to

$$
\hat{y}_{m}=\sum_{n} \frac{H_{m n}}{\sqrt{R_{\eta, m m} A_{n n}}} \hat{x}_{n}+\hat{\eta}_{m}=\sum_{n} \widehat{H}_{m n} \hat{x}_{n}+\hat{\eta}_{m}
$$

where $\hat{\eta}_{m}=\eta_{m} / \sqrt{R_{\eta, m m}}$ such that $\mathrm{E}\left\{\hat{\eta}_{m} \hat{\eta}_{p}\right\}=\delta_{m p}$. This form reveals that the system capacity will depend entirely on the behavior of the effective channel coefficients $\widehat{H}_{m n}$, although 
writing the capacity in the form of (15) without resorting to matrix manipulations is difficult (since $\widehat{H}_{m n} \neq 0$ for $m \neq n$ ). One thing we immediately observe is that the normalization by the eigenvalues $A_{n n}$ and $R_{\eta, m m}$ may lead to a situation where unbounded capacity results.

This unbounded capacity can be tied directly to the phenomenon referred to as supergain [19]-[22]. Physically, certain transmit eigenfunctions can be associated with very small radiated power (small eigenvalues $A_{n n}$ ) but can enable very high directional gain. Because the total radiated power is limited in this formulation, such excitations may be highly desireable because they focus their radiated power into the strong communication modes. Similarly, at the receiver supergain modes characterized by a small noise variance $R_{\eta, m m}$ can be exploited to favorably select the signal directions while suppressing noise from other directions. Unfortunately, achieving supergain is considered impractical since it is associated with difficulties such as narrow operating bandwidth, high ohmic loss, extreme sensitivity to the excitation or receive weighting, and very high gradients of the current or weighting function across the volume.

While we may not be able to define an absolute capacity bound for this communication model, we can define a practical bound. It has recently been shown that the impact of supergain can be limited simply by introducing loss and thermal noise into the models for the transmitter and receiver, respectively [22]. Assuming that all transmit eigenfunctions encounter the same loss resistance $R_{L}$, we can introduce this loss by modifying the eigenvalues $A_{n n}$ as

$$
\widehat{A}_{n n}=A_{n n}+R_{L}=A_{n n}+A_{\max }(1 / \mu-1)
$$

where $\mu=A_{\max } /\left(A_{\max }+R_{L}\right)$ is the radiation efficiency of the mode with the largest eigenvalue $A_{\max }$. Similarly, if thermal noise (due, for example, to a front-end amplifier or resistive loss) with a variance of $R_{T}$ is introduced for each receive eigenfunction, modified noise eigenvalues can be written as

$$
\widehat{R}_{\eta, m m}=R_{\eta, m m}+R_{T}=R_{\eta, m m}+R_{\eta, \max } / \mathrm{INR}
$$

where INR is the interference-to-noise ratio of the mode with the largest noise variance $R_{\eta, \max }$. If the definitions of (34) and (35) are used in (33) in place of $A_{n n}$ and $R_{\eta, m m}$, respectively, increased loss or thermal noise levels will reduce the amount of supergain. Mathematically, these additions eliminate the very small eigenvalues associated with supergain weighting.

With this regularization, we assume that the water-filling capacity solution will select a finite set of communication modes which will lead to bounded capacity. We can then resort to matrix notation to express the system capacity as

$$
C=\max _{\widehat{\mathbf{R}}_{x}} \log _{2} \operatorname{det}\left[\hat{\mathbf{H}} \widehat{\mathbf{R}}_{x} \hat{\mathbf{H}}^{\dagger}+\mathbf{I}\right]
$$

where $\widehat{\mathbf{R}}_{x}=\mathrm{E}\left\{\hat{\mathbf{x}} \hat{\mathbf{x}}^{\dagger}\right\}$. The optimal covariance $\widehat{\mathbf{R}}_{x}$ can be determined using the water-filling solution under the constraint $\operatorname{Tr}\left(\widehat{\mathbf{R}}_{x}\right) \leq P_{T}[$ see (28)].
This capacity formulation is directly related to the analyses provided in [12] and [13]. Specifically, these prior studies represent the transmission in terms of eigenfunctions of the radiation operator, and therefore constraining the basis coefficients will constrain the radiated power (as opposed to the currents). This results in supergain solutions and unbounded capacity, although in prior studies the supergain is restricted by limiting the number of basis functions used in the expansion. While this is a reasonable approach, it is important to recognize that the level of supergain for a linear combination of modes can be acceptable even if a small amount of the energy is devoted to a mode characterized by a high degree of supergain. Therefore, using a complete basis but limiting the impact of supergain by practical considerations (such as loss and noise) provides a method for achieving the optimal performance under practical constraints that can be directly tied to real materials and system architectures.

Finally, we emphasize that when investigating the transmit currents or receive weights with their associated radiation patterns, it is typically desired to view the physical characteristics of the actual communication modes rather than the basis functions $\boldsymbol{\rho}_{m}\left(\mathbf{r}_{\mathrm{r}}\right)$ and $\boldsymbol{\tau}_{n}\left(\mathbf{r}_{\mathrm{t}}\right)$ which for this formulation are eigenfunctions of the noise covariance and radiation operators, respectively. Assuming that only a finite basis is required to perform the capacity analysis, we can express the SVD of the effective channel matrix as $\widehat{\mathbf{H}}=\widehat{\mathbf{U}} \widehat{\mathbf{S}} \widehat{\mathbf{V}}^{\dagger}$. The $q$ th communication mode has transmit coefficients $\hat{\mathbf{x}}_{q}=\hat{\mathbf{v}}_{q}$ representing the $q$ th column of $\hat{\mathbf{V}}$, indicating that the actual current weight vector is given as $\mathbf{x}=\widehat{\mathbf{A}}^{-1 / 2} \hat{\mathbf{v}}_{q}$, where $\widehat{\mathbf{A}}$ is the diagonal matrix with entries $\widehat{A}_{n n}$. A similar analysis can be performed at the receiver.

\section{B. Model Problem}

A simple problem where eigenfunctions can be determined in closed form consists of currents flowing in the axial $(z)$ direction on the surface of a circular cylinder of radius $a_{\mathrm{t}}$. The received field is sampled on the surface of an identically-oriented receive cylinder of radius $a_{\mathrm{r}}$. Equation (3) can then be expressed in cylindrical coordinates (for $\theta=\pi / 2$ ) as $\psi\left(\mathbf{r}^{\prime}, \Omega\right)=$ $e^{-j k \rho^{\prime} \cos \left(\phi-\phi^{\prime}\right)}$. The transmit and receive eigenfunctions for this problem are

$$
\tau_{n}\left(\mathbf{r}_{\mathrm{t}}\right)=\frac{e^{j n \phi_{\mathrm{t}}}}{\sqrt{2 \pi a_{\mathrm{t}}}} \quad \rho_{m}\left(\mathbf{r}_{\mathrm{r}}\right)=\frac{e^{j m \phi_{\mathrm{r}}}}{\sqrt{2 \pi a_{\mathrm{r}}}}
$$

for $-\infty<m, n<\infty$, which can be readily demonstrated by substitution of these functions into (4) and (32). The eigenvalues become

$$
A_{n n}=\frac{\pi a_{\mathrm{t}}}{Z_{0}} J_{n}^{2}\left(k a_{\mathrm{t}}\right) \quad R_{\eta, m m}=2 \pi a_{\mathrm{r}} \sigma_{\eta}^{2} J_{m}^{2}\left(k a_{\mathrm{r}}\right)
$$

where $J_{n}(\cdot)$ is the Bessel function of the first kind of order $n$. Finally, using the LOS propagation described in (16), the normalized channel has coefficients

$$
\widehat{H}_{m n}=\frac{2 Z_{0}}{\sigma_{\eta}} \beta j^{(n-m)} .
$$

Clearly, the resulting matrix $\widehat{\mathbf{H}}$ has unity rank, which is consistent with the LOS propagation model. However, the Frobenius 

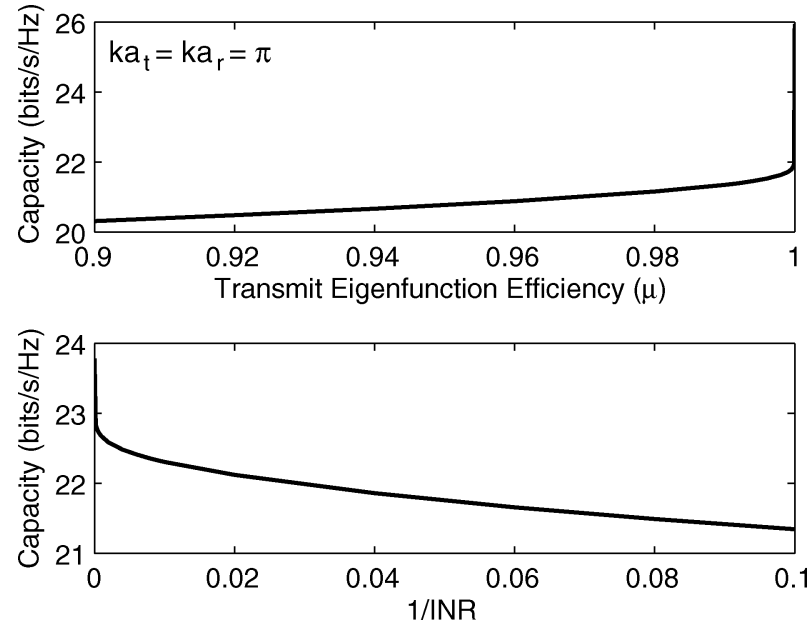

Fig. 5. Capacity versus transmit efficiency for INR $=10 \mathrm{~dB}$ and versus $1 / \mathrm{INR}$ for an efficiency of $\mu=0.99$ for transmit current and field reception on the surface of circular cylinders with radii $a_{\mathrm{t}}=a_{\mathrm{r}}=\lambda / 2$.

norm of the matrix is unbounded as $|m|,|n| \rightarrow \infty$, which indicates that the capacity of this system is unbounded. If, however, the eigenvalues are modified as indicated in (34) and (35), the capacity will be bounded. Fig. 5 plots the capacity as a function of transmit eigenfunction efficiency $\mu$ at an INR of $10 \mathrm{~dB}$ for cylinders with $a_{\mathrm{t}}=a_{\mathrm{r}}=\lambda / 2$. The figure also shows the capacity as a function of 1/INR (to emphasize the behavior at low thermal noise) for an efficiency of $\mu=0.99$. These curves emphasize the dramatic impact of supergain on the performance, including unbounded capacity growth as the loss or noise is reduced to zero.

It is also possible to examine this case for thermal noise where supergain at the receiver does not contribute to increased capacity. In this case, the analysis changes slightly, although the final conclusion of infinite capacity remains valid unless the transmit supergain is restricted as discussed.

\section{Numerical Approximation of Eigenfunctions}

For more general transmit currents and receive volumes, we must again resort to a numerical approximation to the eigenfunctions. Substitution of (18) into (27) produces the matrix

$$
\mathbf{A}=\mathbf{C}^{\dagger} \widetilde{\mathbf{A}} \mathbf{C}
$$

where $\widetilde{A}_{n q}$ is computed from (27) with sub-basis functions replacing the basis functions. We can also express $\mathbf{R}_{\eta}=\mathbf{B}^{\dagger} \widetilde{\mathbf{R}}_{\eta} \mathbf{B}$ where $\widetilde{\mathbf{R}}_{\eta}$ is computed from (32) with the sub-basis functions replacing the basis functions. Since $\widetilde{\mathbf{A}}$ and $\widetilde{\mathbf{R}}_{\eta}$ are Hermitian matrices, their eigenvalue decompositions (EVD) can be expressed as $\widetilde{\mathbf{A}}=\widetilde{\boldsymbol{\xi}}_{A} \widetilde{\boldsymbol{\Lambda}}_{A} \widetilde{\boldsymbol{\xi}}_{A}^{\dagger}$ and $\widetilde{\mathbf{R}}_{\eta}=\widetilde{\boldsymbol{\xi}}_{\eta} \widetilde{\boldsymbol{\Lambda}}_{\eta} \widetilde{\boldsymbol{\xi}}_{\eta}^{\dagger}$, where the eigenvector matrices $\widetilde{\boldsymbol{\xi}}_{A}$ and $\widetilde{\boldsymbol{\xi}}_{\eta}$ are unitary and the diagonal eigenvalue matrices $\widetilde{\Lambda}_{A}$ and $\widetilde{\Lambda}_{\eta}$ are real. We must therefore choose $\mathbf{B}=\widetilde{\boldsymbol{\xi}}_{\eta}$ and $\mathbf{C}=\widetilde{\boldsymbol{\xi}}_{A}$ to diagonalize $\mathbf{R}_{\eta}$ and $\mathbf{A}$, with the desired eigenvalues given as $A_{n n}=\widetilde{\Lambda}_{A, n n}$ and $R_{\eta, m m}=\widetilde{\Lambda}_{\eta, m m}$.

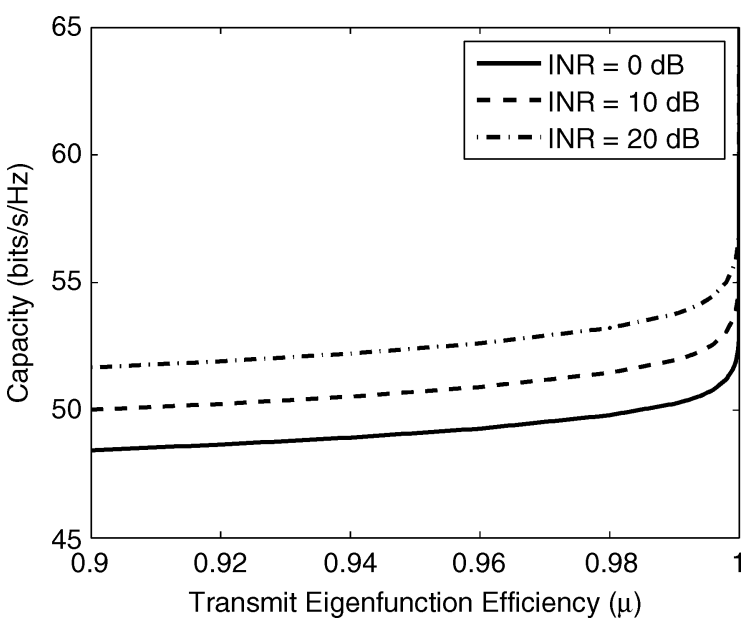

Fig. 6. Numerically computed capacity versus transmit effiency for three different INR values using the modified channel description $(\Delta x=\Delta y=1 \lambda)$.

The resulting eigenvalues can be regularized using the definitions in (34) and (35) for suppression of supergain. Then, computing $\widetilde{\mathbf{H}}$ for the sub-basis functions as in (19) and using the definition of $\widehat{H}_{m n}$ in (33) allows us to write

$$
\widehat{\mathbf{H}}=\widehat{\mathbf{R}}_{\eta}^{-1 / 2} \widetilde{\boldsymbol{\xi}}_{\eta}^{\dagger} \widetilde{\mathbf{H}} \widetilde{\boldsymbol{\xi}}_{A} \widehat{\mathbf{A}}^{-1 / 2}
$$

We finally can compute the system capacity for this channel matrix using (36).

We will again use the basis functions and channel realization discussed in Section III-C. Equation (23) remains valid for the channel matrix $\widetilde{\mathbf{H}}$. Equation (27) for this sub-basis yields

$$
\widetilde{A}_{n q}=\frac{b^{2}}{2 Z_{0}} \int_{0}^{2 \pi} d \phi_{\mathrm{t}} \gamma^{*}\left(\mathbf{r}_{\mathrm{t} n}, \Omega_{\mathrm{t}}\right) \gamma\left(\mathbf{r}_{\mathrm{t} q}, \Omega_{\mathrm{t}}\right) .
$$

For the identical transmit and receive apertures used here and assuming $p_{\eta}\left(\Omega_{\mathrm{r}}\right)=\sigma_{\eta}^{2}, \widetilde{R}_{\eta, m p}=2 Z_{0} \sigma_{\eta}^{2} \widetilde{A}_{m p}$. To specify the system SNR, we compute each quantity for a single sub-basis function as in Section III-C and define the system SNR as

$$
\mathrm{SNR}=\frac{P_{T}\left|\widetilde{H}_{11}\right|^{2}}{\widetilde{A}_{11} \widetilde{R}_{\eta, 11}}
$$

Once again, the value of $\sigma_{\eta}^{2}$ required to obtain an SNR of $20 \mathrm{~dB}$ is computed, and this value is held constant as the number of sub-basis functions increases.

Fig. 6 plots the capacity versus the transmit effiency for an aperture with $\Delta x=\Delta y=1 \lambda$ and three different values of INR. The computation has been performed using $N=20$ basis functions per dimension based upon a convergence study. The results confirm the findings associated with Fig. 5, showing bounded capacity only for non-zero transmit loss and receive thermal noise. More importantly, this example demonstrates application of the approach to general geometries and channel propagation environments. 


\section{CONCLUSION}

This paper has presented a general framework for determining the available capacity of continuous-space electromagnetic channels. The approach expands the transmit antenna currents and receive antenna weights using basis functions, and determines the optimal functional form for these quantities for the electromagnetic propagation channel. Prior analyses of this problem fit within this general framework, with different approaches effectively corresponding to using basis functions consisting of eigenfunctions of different operators. These solutions are directly linked to the constraint on the transmit power and the assumptions regarding the correlation of the receiver noise. The advantage of this general formulation is that it highlights the key differences between the existing analyses and provides insight into the interpretation of the results. Furthermore, the insight enabled by the framework allows actually mixing the assumptions (such as constraining the current magnitude combined with spatially correlated receiver noise). Finally, the fact that the method produces the aperture distributions and radiation patterns implies that it has the potential to be used as part of a more comprehensive antenna synthesis approach.

\section{REFERENCES}

[1] G. J. Foschini and M. J. Gans, "On limits of wireless communications in a fading environment when using multiple antennas," Wireless Personal Commun., vol. 6, pp. 311-335, Mar. 1998.

[2] G. G. Raleigh and J. M. Cioffi, "Spatio-temporal coding for wireless communication," IEEE Trans. Commun., vol. 46, pp. 357-366, Mar. 1998.

[3] C. C. Martin, J. H. Winters, and N. R. Sollenberger, "Multiple-input multiple-output (MIMO) radio channel measurements," in Proc. IEEE Antennas and Propag. Society Int. Symp., Boston, MA, Jul. 2001, vol. 1, pp. 418-421.

[4] J. W. Wallace, M. A. Jensen, A. L. Swindlehurst, and B. D. Jeffs, "Experimental characterization of the MIMO wireless channel: Data acquisition and analysis," IEEE Trans. Wireless Commun., vol. 2, pp. 335-343, Mar. 2003.

[5] G. D. Golden, C. J. Foschini, R. A. Valenzuela, and P. W. Wolniansky, "Detection algorithm and initial laboratory results using V-BLAST space-time communication architecture," Electron. Lett., vol. 35, pp. 14-16, Jan. 1999.

[6] V. Tarokh, N. Seshadri, and A. R. Calderbank, "Space-time codes for high data rate wireless communication: Performance criterion and code construction," IEEE Trans. Inf. Theory, vol. 44, pp. 744-765, Mar. 1998.

[7] Z. Liu, G. B. Giannakis, S. Barbarossa, and A. Scaglione, "Transmit antennae space-time block coding for generalized OFDM in the presence of unknown multipath," IEEE J. Select. Areas Commun., vol. 18, pp. 1352-1364, Jul. 2001

[8] R. A. Andrews, P. P. Mitra, and R. deCarvalho, "Tripling the capacity of wireless communications using electromagnetic polarization," $\mathrm{Na}$ ture, vol. 409, pp. 316-318, Jan. 2001.

[9] D. A. Miller, "Communicating with waves between volumes: Evaluating orthogonal spatial channels and limits on coupling strengths," Appl. Opt., vol. 39, pp. 1681-1699, Apr. 2000.

[10] J. W. Wallace and M. A. Jensen, "Intrinsic capacity of the MIMO wireless channel," in Proc. IEEE Antennas and Propag. Society Int. Symp., San Antonio, TX, Jun. 2002, vol. 3, pp. 198-201.

[11] L. Hanlen and M. Fu, "Wireless communication systems with spatial diversity: A volumetric model," IEEE Trans. Wireless Commun., vol. 5, pp. 133-142, Jan. 2006.

[12] S. Nordebo, M. Gustafsson, and G. Kristensson, "On the capacity of the free space antenna channel," in Proc. IEEE Antennas and Propag. Society Int. Symp., Albuquerque, NM, Jul. 2006, pp. 3105-3108.
[13] M. D. Migliore, "On the role of the number of degrees of freedom of the field in MIMO channels," IEEE Trans. Antennas Propag., vol. 54, pp. 620-628, Feb. 2006.

[14] J. A. Kong, Electromagnetic Wave Theory. New York: Wiley, 1990.

[15] A. W. Naylor and G. R. Sell, Eds., Linear Operator Theory in Engineering and Science. Berlin, Germany: Springer-Verlag, 1982.

[16] T. M. Cover and J. A. Thomas, Elements of Information Theory. New York: Wiley, 1991

[17] J. W. Wallace and M. A. Jensen, "Modeling the indoor MIMO wireless channel," IEEE Trans. Antennas Propag., vol. 50, pp. 591-599, May 2002

[18] A. A. M. Saleh and R. A. Valenzuela, "A statistical model for indoor multipath propagation," IEEE J. Select. Areas Commun., vol. SAC-5, pp. 128-137, Feb. 1987.

[19] Y. T. Lo, S. W. Lee, and Q. H. Lee, "Optimization of directivity and signal-to-noise ratio of an arbitrary antenna array," Proc. IEEE, vol. 54, pp. 1033-1045, Aug. 1966.

[20] S. M. Sanzgiri and J. K. Butler, "Constrained optimization of the performance indices of arbitrary array antennas," IEEE Trans. Antennas Propag., vol. AP-19, pp. 493-498, Jul. 1971.

[21] M. Uzsoky and L. Solymar, "Theory of super-directive linear antennas," Acta Physica Hungarica, vol. 6, no. 2, pp. 185-205, 1956.

[22] N. W. Bikhazi and M. A. Jensen, "The relationship between antenna loss and superdirectivity in MIMO systems," IEEE Trans. Wireless Commun., vol. 6, pp. 1796-1802, May 2007.

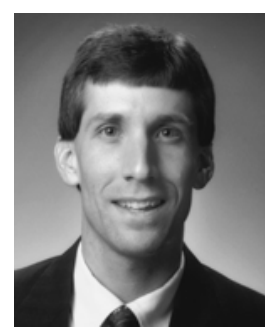

Michael A. Jensen (S'93-M'95-SM'01-F'08) received the B.S. (summa cum laude) and M.S. degrees in electrical engineering from Brigham Young University (BYU), Provo, UT, in 1990 and 1991, respectively, and the Ph.D. in electrical engineering from the University of California, Los Angeles (UCLA), in 1994.

From 1989 to 1991, he was a Graduate Researcher in the Lasers and Optics Laboratory at BYU, during which time he received a National Science Foundation Graduate Fellowship. From 1991 to 1994, he was a Graduate Student Researcher in the Antenna Laboratory at UCLA. Since 1994, he has been in the Electrical and Computer Engineering Department at BYU where he is currently a Professor and Department Chair. His research interests include antennas and propagation for communications, microwave circuit design, numerical electromagnetics, and optical fiber communications.

Dr. Jensen is a member of Eta Kappa Nu and Tau Beta Pi. He currently chairs the Joint Meetings Committee for the IEEE Antennas and Propagation Society and previously served as a member of the Administrative Committee for this same society and as an Associate Editor for the IEEE TRANSACTIONS ON ANTENNAS AND PROPAGATION. He has also served as Vice-Chair or Technical Program Chair for four different symposia. He received the H. A. Wheeler Applications Prize Paper Award from the IEEE Antennas and Propagation Society in 2002, and the Best Student Paper Award presented at the 1994 IEEE International Symposium on Antennas and Propagation.

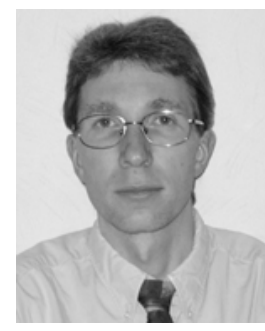

Jon W. Wallace (S'99-M'03) received the B.S. (summa cum laude) and Ph.D. degrees in electrical engineering from Brigham Young University (BYU), Provo, UT, in 1997 and 2002, respectively.

From 1995 to 1997, he worked as an Associate of Novell, Inc., Provo. During 1997, he was a Member of Technical Staff for Lucent Technologies, Denver, CO. He received the National Science Foundation Graduate Fellowship in 1998 and worked as a Graduate Research Assistant at BYU until 2002. From 2002 to 2003, he was with the Mobile Communications Group, Vienna University of Technology, Vienna, Austria, pursuing collaborative research in the area of wireless channel measurement and modeling. From 2003 to 2006, he was a Research Associate with the BYU Wireless Communications Laboratory, developing platforms for wideband MIMO channel sounding and real-time space-time coding. He is currently an Assistant Professor of electrical engineering at Jacobs University Bremen, Bremen, Germany. His current research interests include wireless channel sounding and modeling, MIMO communications, cognitive radio, and UWB and frequency-agile systems. 